\title{
Analysis of the Conformally Flat Approximation for Binary Neutron Star Initial Conditions
}

\author{
In-Saeng Suh, ${ }^{1}$ Grant J. Mathews, ${ }^{2}$ J. Reese Haywood, ${ }^{2}$ and N. Q. Lan ${ }^{2,3,4}$ \\ ${ }^{1}$ Center for Astrophysics, Department of Physics and Center for Research Computing, University of Notre Dame, \\ Notre Dame, IN 46556, USA \\ ${ }^{2}$ Center for Astrophysics, Department of Physics, University of Notre Dame, Notre Dame, IN 46556, USA \\ ${ }^{3}$ Hanoi National University of Education, 136 Xuan Thuy, Hanoi, Vietnam \\ ${ }^{4}$ Joint Institute for Nuclear Astrophysics (JINA), University of Notre Dame, Notre Dame, IN 46556, USA
}

Correspondence should be addressed to Grant J. Mathews; gmathews@nd.edu

Received 19 September 2016; Accepted 30 November 2016; Published 9 January 2017

Academic Editor: Ignazio Licata

Copyright (C) 2017 In-Saeng Suh et al. This is an open access article distributed under the Creative Commons Attribution License, which permits unrestricted use, distribution, and reproduction in any medium, provided the original work is properly cited.

\begin{abstract}
The spatially conformally flat approximation (CFA) is a viable method to deduce initial conditions for the subsequent evolution of binary neutron stars employing the full Einstein equations. Here we analyze the viability of the CFA for the general relativistic hydrodynamic initial conditions of binary neutron stars. We illustrate the stability of the conformally flat condition on the hydrodynamics by numerically evolving 100 quasicircular orbits. We illustrate the use of this approximation for orbiting neutron stars in the quasicircular orbit approximation to demonstrate the equation of state dependence of these initial conditions and how they might affect the emergent gravitational wave frequency as the stars approach the innermost stable circular orbit.
\end{abstract}

\section{Introduction}

The epoch of gravitational wave astronomy has now begun with the first detection $[1,2]$ of the merger of binary black holes by Advanced LIGO [3]. Now that the first ground based gravitational wave detection has been achieved, observations of binary neutron star mergers should soon be forthcoming. This is particularly true as other second generation observatories such as Advanced VIRGO [4] and KAGRA [5] will soon be online. In addition to binary black holes, neutron star binaries are thought to be among the best candidate sources gravitational radiation $[6,7]$. The number of such systems detectable by Advanced LIGO is estimated [7-14] to be of order several events per year based upon observed close binary-pulsar systems $[15,16]$. There is a difference between neutron star mergers and black hole mergers; however, in that neutron star mergers involve the complex evolution of the matter hydrodynamic equations in addition to the strong gravitational field equations. Hence, one must carefully consider both the hydrodynamic and field evolution of these systems.
To date there have been numerous attempts to calculate theoretical templates for gravitational waves from compact binaries based upon numerical and/or analytic approaches (see, e.g., [17-26]). However, most approaches utilize a combination of post-Newtonian (PN) techniques supplemented with quasicircular orbit calculations and then applying full GR for only the last few orbits before disruption. In this paper we analyze the hydrodynamic evolution in the spatially conformally flat metric approximation (CFA) as a means to compute stable initial conditions beyond the range of validity of the PN regime, that is, near the last stable orbits. We establish the numerical stability of this approach based upon many orbit simulations of quasicircular orbits. We show that one must follow the stars for several orbits before a stable quasicircular orbit can be achieved. We also illustrate the equation of state (EoS) dependence of the initial conditions and associated gravitational wave emission.

When binary neutron stars are well separated, the postNewtonian (PN) approximation is sufficiently accurate [27]. In the PN scheme, the stars are often treated as point masses, either with or without spin. At third order, for example, it has 
been estimated [28-30] that the error due to assuming the stars are point masses is less than one orbital rotation [28] over the $\sim 16,000$ cycles that pass through the LIGO detector frequency band [7]. Nevertheless, it has been noted in many works [25, 31-42] that relativistic hydrodynamic effects might be evident even at the separation $(\sim 10-100 \mathrm{~km})$ relevant to the LIGO window.

Indeed, the templates generated by $\mathrm{PN}$ approximations, unless carried out to fifth and sixth order [28, 29], may not be accurate unless the finite size and proper fluid motion of the stars are taken into account. In essence, the signal emitted during the last phases of inspiral depends upon the finite size and the equation of state (EoS) through the tidal deformation of the neutron stars and the cut-off frequency when tidal disruption occurs.

Numeric and analytic simulations [43-51] of binary neutron stars have explored the approach to the innermost stable circular orbit (ISCO). While these simulations represent some of the most accurate to date, simulations generally follow the evolution for a handful of orbits and are based upon initial conditions of quasicircular orbits obtained in the conformally flat approximation. Accurate templates of gravitational radiation require the ability to stably and reliably calculate the orbit initial conditions. The CFA provides a means to obtain accurate initial conditions near the ISCO.

The spatially conformally flat approximation to GR was first developed in detail in [32]. That original formulation, however, contained a mathematical error first pointed out by Flanagan [52] and subsequently corrected in [34]. This error in the solution to the shift vector led to a spurious NS crushing prior to merger. The formalism discussed below is for the corrected equations. Here, we discuss the hydrodynamic solutions as developed in [31-34, 53, 54]. This CFA formalism includes much of the nonlinearity inherent in GR and leads set of coupled, nonlinear, elliptic field equations that can be evolved stably. We also note that an alternative spectral method solution to the CFA configurations was developed by $[55,56]$, and approaches beyond the CFA have also been proposed [48]. However, our purpose here is to clarify the viability of the hydrodynamic solution without the imposition of a Killing vector or special symmetry. This approach is the most adaptable, for example, to general initial conditions such as that of arbitrarily elliptical orbits and/or arbitrarily spinning neutron stars.

Here, we summarize the original CFA approach and associated general relativistic hydrodynamics formalism developed in $[32,34,53,54]$ and illustrate that it can produce stable initial conditions anywhere between the post-Newtonian to ISCO regimes. We quantify how long this method takes to converge to quasiequilibrium and demonstrate the stability by subsequently integrating up to $\sim 100$ orbits for a binary neutron star system. We also analyze the EoS dependence of these quasicircular initial orbits and show how these orbits can be used to make preliminary estimates [57] of the gravitational wave signal for the initial conditions.

This paper is organized as follows. In Section 2 the basic method is summarized and in Section 3 a number of code tests are performed in the quasiequilibrium circular orbit limit to demonstrate the stability of the technique. The EoS dependence of the initial conditions and associated gravitational wave frequency are analyzed in Section 4. Conclusions are presented in Section 5.

\section{Method}

2.1. Field Equations. The solution of the field equations and hydrodynamic equations of motion were first solved in three spatial dimensions and explained in detail in the 1990s in [31, $32]$ and subsequently further reviewed in $[53,58]$. Here, we present a brief summary to introduce the variables relevant to the present discussion.

One starts with the slicing of space-time into the usual one-parameter family of hypersurfaces separated by differential displacements in a time-like coordinate as defined in the $(3+1)$ ADM formalism $[59,60]$.

In Cartesian $x, y, z$ isotropic coordinates, proper distance is expressed as

$$
d s^{2}=-\left(\alpha^{2}-\beta_{i} \beta^{i}\right) d t^{2}+2 \beta_{i} d x^{i} d t+\phi^{4} \delta_{i j} d x^{i} d x^{j},
$$

where the lapse function $\alpha$ describes the differential lapse of proper time between two hypersurfaces. The quantity $\beta_{i}$ is the shift vector denoting the shift in space-like coordinates between hypersurfaces. The curvature of the metric of the 3-geometry is described by a position-dependent conformal factor $\phi^{4}$ times a flat-space Kronecker delta $\left(\gamma_{i j}=\phi^{4} \delta_{i j}\right)$. This conformally flat condition (together with the maximal slicing gauge, $\operatorname{tr}\left\{K_{i j}\right\}=0$ ) requires [60]

$$
2 \alpha K_{i j}=D_{i} \beta_{j}+D_{j} \beta_{i}-\frac{2}{3} \delta_{i j} D_{k} \beta^{k}
$$

where $K_{i j}$ is the extrinsic curvature tensor and $D_{i}$ are 3-space covariant derivatives. This conformally flat condition on the metric provides a numerically valid initial solution to the Einstein equations. The vanishing of the Weyl tensor for a stationary system in three spatial dimensions guarantees that a conformally flat solution to the Einstein equations exists.

One consequence of this conformally flat approximation to the three-metric is that the emission of gravitational radiation is not explicitly evolved. Nevertheless, one can extract the gravitational radiation signal and the back reaction via a multipole expansion $[32,61]$. An application to the determination of the gravitational wave emission from the quasicircular orbits computed here is given in [57]. The advantage of this approximation is that conformal flatness stabilizes and simplifies the solution to the field equations.

As a third gauge condition, one can choose separate coordinate transformations for the shift vector and the hydrodynamic grid velocity to separately minimize the field and matter motion with respect to the coordinates. This set of gauge conditions is key to the present application. It allows one to stably evolve up to hundreds and even thousands of binary orbits without the numerical error associated with the frequent advocating of fluid through the grid.

Given a distribution of mass and momentum on some manifold, then one first solves the constraint equations of general relativity at each time for a fixed distribution of 
matter. One then evolves the hydrodynamic equations to the next time step. Thus, at each time slice a solution to the relativistic field equations and information on the hydrodynamic evolution is obtained.

The solutions for the field variables $\phi, \alpha$, and $\beta^{i}$ reduce to simple Poisson-like equations in flat space. The Hamiltonian constraint [60] is used to solve for the conformal factor $\phi[32$, 62]

$$
\nabla^{2} \phi=-2 \pi \phi^{5}\left[W^{2}(\rho(1+\epsilon)+P)-P+\frac{1}{16 \pi} K_{i j} K^{i j}\right] .
$$

In the Newtonian limit, the RHS is dominated [32] by the proper matter density $\rho$, but in strong fields and compact neutron stars there are also contributions from the internal energy density $\epsilon$, pressure $P$, and extrinsic curvature. The source is also significantly enhanced by the generalized curved-space Lorentz factor $W$

$$
W=\alpha U^{t}=\left[1+\frac{\sum U_{i}^{2}}{\phi^{4}}\right]^{1 / 2}
$$

where $U^{t}$ is the time component of the relativistic four velocity and $U_{i}$ are the covariant spatial components. This factor, $W$, becomes important near the last stable orbit as the specific kinetic energy of the stars rapidly increases.

In a similar manner $[32,62]$, the Hamiltonian constraint, together with the maximal slicing condition, provides an equation for the lapse function,

$$
\begin{aligned}
& \nabla^{2}(\alpha \phi)=2 \pi \alpha \phi^{5}\left[3 W^{2}[\rho(1+\epsilon)+P]-2 \rho(1+\epsilon)\right. \\
& \left.+3 P+\frac{7}{16 \pi} K_{i j} K^{i j}\right] .
\end{aligned}
$$

Finally, the momentum constraints yields [60] an elliptic equation for the shift vector $[34,52]$,

$$
\nabla^{2} \beta^{i}=\frac{\partial}{\partial x^{i}}\left(\frac{1}{3} \nabla \cdot \beta\right)+4 \pi \rho_{3}^{i},
$$

where

$$
\begin{aligned}
\rho_{3}^{i}= & 4 \alpha \phi^{4} S_{i} \\
& +\frac{1}{4 \pi} \frac{\partial \ln \left(\alpha / \phi^{6}\right)}{\partial x^{j}}\left(\frac{\partial \beta^{i}}{\partial x^{j}}+\frac{\partial \beta^{j}}{\partial x^{i}}-\frac{2}{3} \delta_{i j} \frac{\partial \beta^{k}}{\partial x^{k}}\right) .
\end{aligned}
$$

Here $S_{i}$ are the spatial components of the momentum density one-form as defined below.

We note that, in early applications of this approach, the source for the shift vector contained a spurious term due to an incorrect transformation between contravariant and covariant forms of the momentum density as was pointed out in $[34,52]$. As illustrated in those papers, this was the main reason why early hydrodynamic calculations induced a controversial additional compression on stars causing them to collapse to black holes prior to inspiral [31]. This problem no longer exists in the formulation summarized here.
2.2. Relativistic Hydrodynamics. To solve for the fluid motion of the system in curved-space time it is convenient to use an Eulerian fluid description [63]. One begins with the perfect fluid stress-energy tensor in the Eulerian observer rest frame,

$$
T_{\mu \nu}=P g_{\mu \nu}+(\rho(1+\epsilon)+P) U_{\mu} U_{\nu}
$$

where $U_{\mu}$ is the relativistic four velocity one-form.

By introducing the usual set of Lorentz contracted state variables it is possible to write the relativistic hydrodynamic equations in a form which is reminiscent of their Newtonian counterparts [63]. The hydrodynamic state variables are the coordinate baryon mass density

$$
D=W \rho
$$

the coordinate internal energy density

$$
E=W \rho \epsilon
$$

the spatial three velocity

$$
V^{i}=\alpha \frac{U_{i}}{\phi^{4} W}-\beta^{i} ;
$$

and the covariant momentum density

$$
S_{i}=(D+E+P W) U_{i}
$$

In terms of these state variables, the hydrodynamic equations in the CFA are as follows: the equation for the conservation of baryon number takes the form

$$
\frac{\partial D}{\partial t}=-6 D \frac{\partial \log \phi}{\partial t}-\frac{1}{\phi^{6}} \frac{\partial}{\partial x^{j}}\left(\phi^{6} D V^{j}\right) .
$$

The equation for internal energy evolution becomes

$$
\begin{aligned}
\frac{\partial E}{\partial t}= & -6(E+P W) \frac{\partial \log \phi}{\partial t}-\frac{1}{\phi^{6}} \frac{\partial}{\partial x^{j}}\left(\phi^{6} E V^{j}\right) \\
& -P\left[\frac{\partial W}{\partial t}+\frac{1}{\phi^{6}} \frac{\partial}{\partial x^{j}}\left(\phi^{6} W V^{j}\right)\right] .
\end{aligned}
$$

Momentum conservation takes the form

$$
\begin{aligned}
\frac{\partial S_{i}}{\partial t}= & -6 S_{i} \frac{\partial \log \phi}{\partial t}-\frac{1}{\phi^{6}} \frac{\partial}{\partial x^{j}}\left(\phi^{6} S_{i} V^{j}\right)-\alpha \frac{\partial P}{\partial x^{i}} \\
& +2 \alpha(D+E+P W)\left(W-\frac{1}{W}\right) \frac{\partial \log \phi}{\partial x^{i}} \\
& +S_{j} \frac{\partial \beta^{j}}{\partial x^{i}}-W(D+E+P W) \frac{\partial \alpha}{\partial x^{i}} \\
& -\alpha W(D+\Gamma E) \frac{\partial \chi}{\partial x^{i}},
\end{aligned}
$$

where the last term in (15) is the contribution from the radiation reaction potential $\chi$ as defined in $[32,57]$. In the construction of quasistable orbit initial conditions, this term is set to zero. Including this term would allow for a calculation of the orbital evolution via gravitational wave emission in the CFA. However, there is no guarantee that this is a sufficiently accurate solution to the exact Einstein equations. Hence, the CFA is useful for the construction of initial conditions. 
2.3. Angular Momentum and Orbital Frequency. In the quasicircular orbit approximation (neglecting angular momentum in the radiation field), this system has a Killing vector corresponding to rotation in the orbital plane. Hence, for these calculations the angular momentum is well defined and given by an integral over the space-time components of the stress-energy tensor [64]; that is,

$$
J^{i j}=\int\left(T^{i 0} x^{j}-T^{j 0} x^{i}\right) d V
$$

Aligning the $z$-axis with the angular momentum vector then gives

$$
J=\int\left(x S^{y}-y S^{x}\right) d V
$$

To find the orbital frequency detected by a distant observer corresponding to a fixed angular momentum we employ a slightly modified derivation of the orbital frequency compared to that of [53]. In asymptotically flat coordinates the angular frequency detected by a distant observer is simply the coordinate angular velocity; that is, one evaluates

$$
\omega \equiv \frac{d \phi}{d t}=\frac{U^{\phi}}{U^{0}}
$$

In the ADM conformally flat $(3+1)$ curved space, our only task is then to deduce $U^{\phi}$ from code coordinates. For this we make a simple polar coordinate transformation keeping our conformally flat coordinates, so

$$
U^{\phi}=\Lambda_{\nu}^{\phi} U^{\nu}=\frac{x U^{y}-y U^{x}}{x^{2}+y^{2}} .
$$

Now, the code uses covariant four velocities, $U_{i}=g_{i \nu} U^{\nu}=$ $\beta_{i} U^{0}+\phi^{4} U^{i}$. This gives $U^{i}=U_{i} \beta_{i}(W / \alpha) / \phi^{4}$. Finally, one must density weight and volume average $\omega$ over the fluid differential volume elements. This gives

$$
\omega=\frac{\int d^{3} x \phi^{2}(D+\Gamma E)\left[(\alpha / W)\left(x U_{y}-y U_{x}\right)-\left(x \beta_{y}-y \beta_{x}\right)\right] /\left(x^{2}+y^{2}\right)}{\int d^{3} x \phi^{6}(D+\Gamma E)} .
$$

This form differs slightly from that of [53] but leads to the similar results.

A key additional ingredient, however, is the implementation of a grid three velocity $V_{G}^{i}$ that minimizes the matter motion with respect to $U_{i}$ and $\beta_{i}$. Hence, the total angular frequency to a distant observer $\omega_{\text {tot }}=\omega+\omega_{G}$, and in the limit of rigid corotation, $\omega_{\text {tot }} \rightarrow \omega_{G}$, where $\omega_{G}=x V^{y}+y V^{x}$.

For the orbit calculations illustrated here we model corotating stars, that is, no spin in the corotating frame. This minimizes matter motion on the grid. However, we note that there is need at the present time of initial conditions for arbitrarily spinning neutron stars and the method described here is equally capable of supplying those initial conditions.

As a practical approach the simulation [32] of initial conditions is best run first with viscous damping in the hydrodynamics for sufficiently long time (a few thousand cycles) to relax the stars to a steady state. Subsequently, one can run with no damping. In the present illustration we examine stars at large separation which are in quasiequilibrium circular orbits and stable hydrodynamic configurations. In the initial relaxation phase the orbits are circularized by damping any radial velocity components. During the evolution, the rigorous conservation of angular momentum is imposed by adjusting the orbital angular velocity in (20) such that (17) remains constant. The simulated orbits described in this work span the time from the last several minutes up to orbit inspiral. Here, we illustrate the stability of the multiple orbit hydrodynamic simulation and examine where the initial conditions for the strong field orbit dynamics computed here deviates from the post-Newtonian regime.

\section{Code Validation}

3.1. Code Tests. To evolve stars at large separation distance it is best [53] to decompose the grid into a high resolution domain with a fine matter grid around the stars and a coarser domain with an extended grid for the fields. Figure 1 shows a schematic of this decomposition from [54].

As noted in [53] it is best to keep the number of zones across each star between 25 and 40 [54]. This keeps the error in the numerics below $0.5 \%$. It has also been pointed out [53] that an artificial viscosity (AV) shock capturing scheme has an advantage over Riemann solvers because only about half as many zones are required to accurately resolve the stars when an $\mathrm{AV}$ scheme is employed compared to a Riemann solver.

The time steps $d t_{n}$ are taken as the minimum of the time step as determined by several conditions. Each condition is also multiplied by a number less than one to accommodate the nonlinear nature of the equations.

The first condition is known as the Courant condition, that is, a search over all zones $i$ for the zone with the minimum sound crossing time:

$$
d t_{1}=\min \left(\frac{d x_{b}^{i}}{C_{s}^{i}}\right)
$$

where $C_{s}^{i}$ is the sound speed in the $i$ th zone.

The Newtonian sound speed is given by the variation of pressure with density. In relativity the wave speed is given instead by the adiabatic derivative of the pressure with respect 


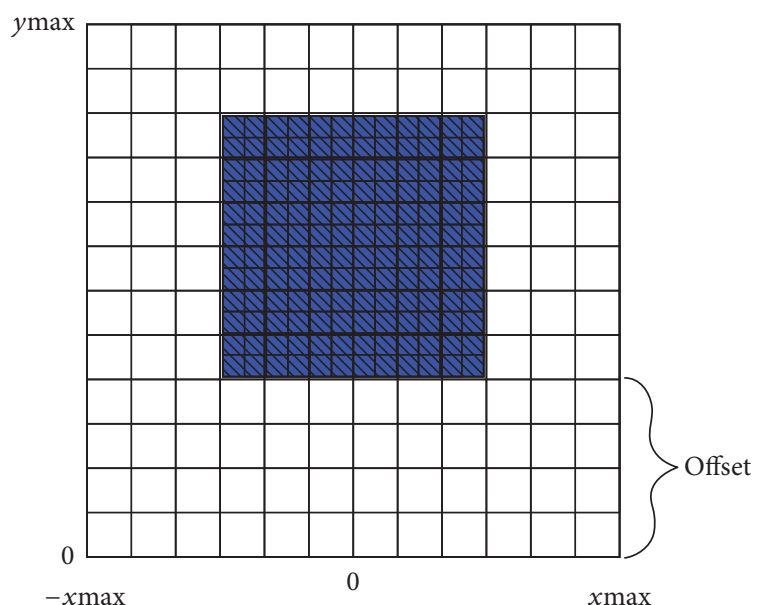

FIGURE 1: Schematic representation of the field and hydrodynamics grid used in the simulations. The inner blue grid represents the higher resolution matter grid and the outer white grid represents the field grid.

to the relativistic inertial density. In terms of relativistic variables the local sound speed in zone $i$ then becomes [53]

$$
C_{s}=\sqrt{\frac{\Gamma_{i}\left(\Gamma_{i}-1\right) E_{i}}{D_{i}+\Gamma_{i} E_{i}}} .
$$

The second condition is a search for the zone with minimum time for material to flow across a zone

$$
d t_{2}=\min \left(\frac{d x_{a}^{i}}{\left|V^{i}\right|}\right) .
$$

This constraint is introduced to ensure stability and accuracy in the numerical advection calculation.

The third condition is introduced to maintain stability of the artificial viscosity algorithm. The viscous equations are analogous to a diffusion equation in four velocity with a diffusion coefficient $D \approx k_{1} d x^{i}\left|\delta U^{i}\right|$, where $\delta U^{i} \equiv U^{i+1}-$ $U^{i}$. We then can define a minimum viscous diffusion time across a zone derived from the stability condition for explicit diffusion equations

$$
d t_{3}=\frac{1}{4} \min \left(\frac{W^{i} d x_{b}^{i}}{\left|\delta U^{i}\right|}\right) .
$$

The time step $d t$ is then assigned to be some fraction (referred to as the Courant parameter) of the minimum of these three conditions

$$
d t=k \min \left(d t_{1}, d t_{2}, d t_{3}\right),
$$

Obviously, smaller values for $k$ increase the accuracy of the calculation but also increase the computation time.

Figure 2 shows a plot of orbital velocity versus time for various values of the Courant parameter. This figure establishes that the routines for the hydrodynamics are stable (e.g., changing the Courant condition has little to effect) as long as $k \leq 0.5$.

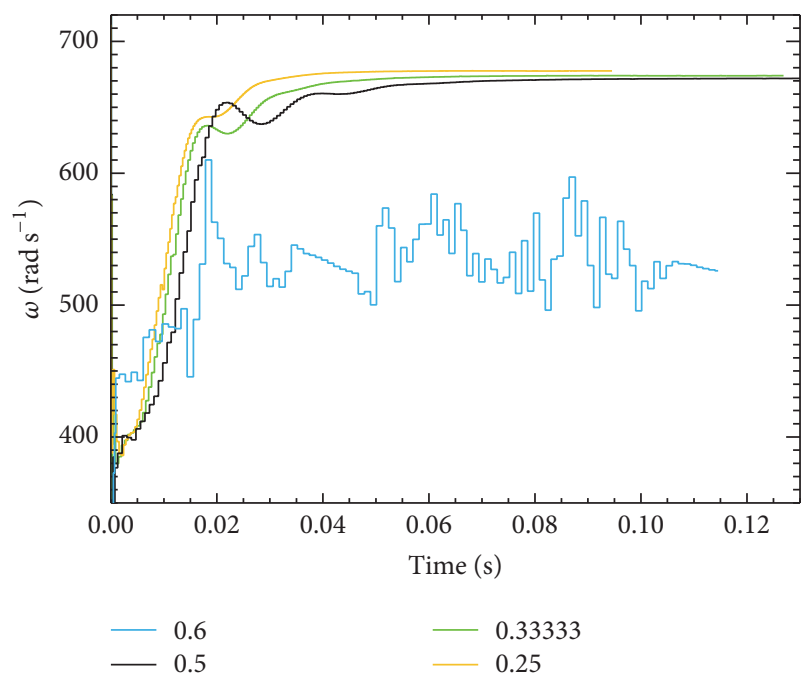

FIgURE 2: Comparison of the orbital angular velocity $\omega$ versus time for different values of the Courant parameter $k$. As can be seen, the simulations with $k=0.25-0.5$ result in stable runs that converge to the same value, implying that a smaller $k$ or equivalently a smaller $\delta t$ is not necessary and would only use extra CPU time. For comparison, we plot a simulation with $k=0.6$ to show that the stability is lost for $k>0.5$.

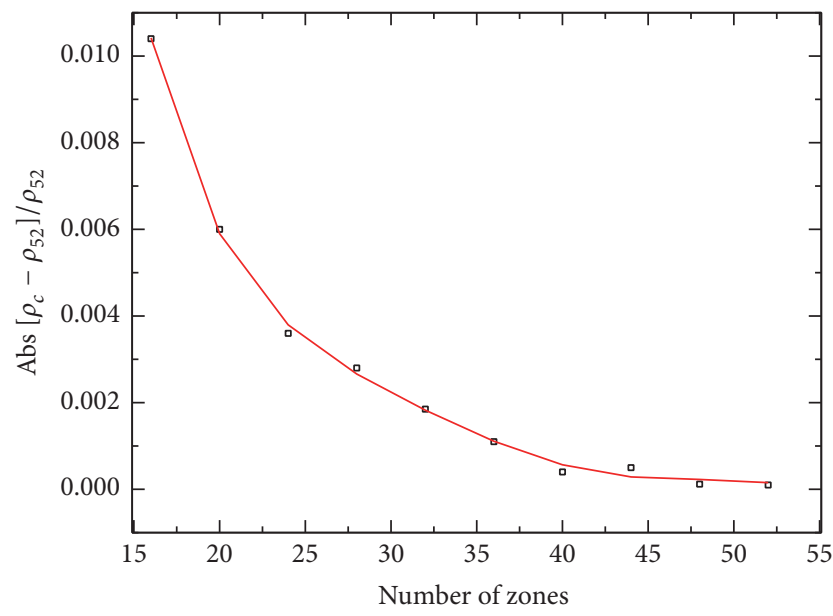

Figure 3: Plot of the error in the central density versus the number of zones across the star. It is clear that there is only a $1 \%$ error with $\approx 15$ zones across the star. Increasing the number of zones across the star so that there are $>35$ zones across the star produces less than a $0.1 \%$ error.

Figure 3 illustrates the difference between the central density $\rho_{c}$ and the central density $\left(\rho_{52}\right)$ at the highest resolution of 52 zones for single isolated stars. This is expressed as the fractional error as a function of the number of zones across a star. This plot was calculated using the relatively soft MW EoS, that is, the zero temperature and zero neutrino chemical potential limit of the EoS that has previously been used to model core-collapse supernovae $[32,53,65]$.

This figure illustrates that here is only a $1 \%$ error in central density with $\approx 15$ zones across the star, while increasing the 


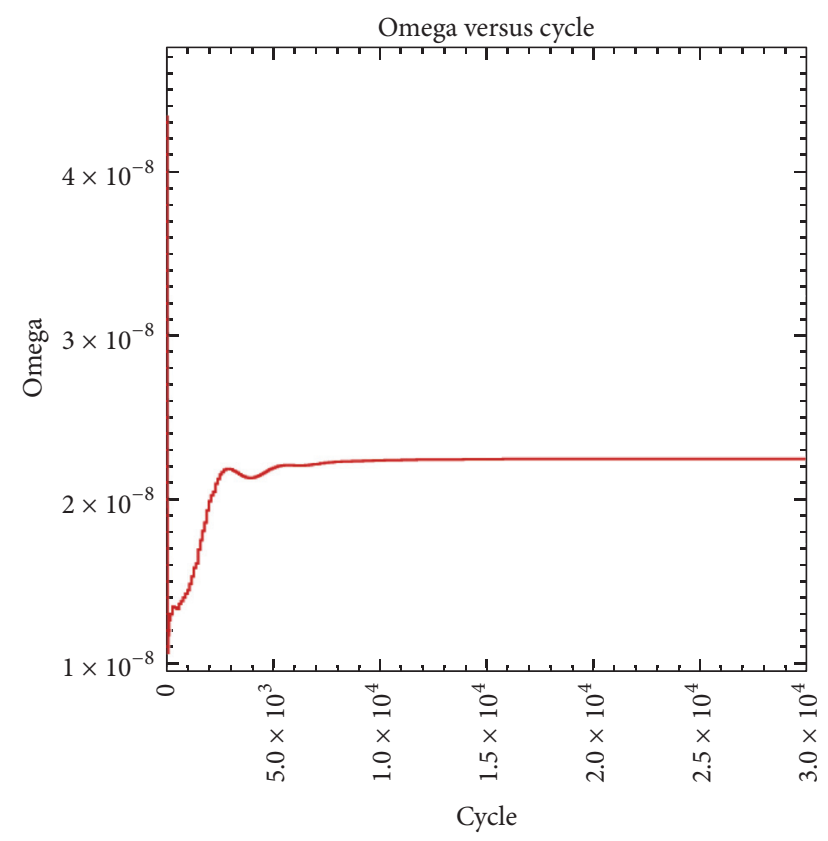

FIGURE 4: Plot of the orbital angular velocity, $\omega$ in geometrized units $\left(\mathrm{cm}^{-1}\right)$ versus cycle number. When $\omega$ stops changing the simulation has reached a circular binary orbit solution. This run was extended to over 30,000 cycles, corresponding to $\approx 20$ orbits.

number of zones across the star to $>35$ produces less than a $0.1 \%$. In the illustrations below we maintain $k=0.5$ and $\approx 25$ zones across each star as the best choice for both speed and accuracy needed to compute stable orbital initial conditions.

3.2. Orbit Stability. As an illustration of the orbit stability Figure 4 shows results from a simulation [54] in which the angular momentum was fixed at $J=2.7 \times 10^{11} \mathrm{~cm}^{2}$ and the Courant parameter set to $k=0.5$. For this orbital calculation the MW EoS was employed and each star was fixed at a baryon mass of $M_{B}=1.54 \mathrm{M}_{\odot}$ and a gravitational mass in isolation of $M_{G}=1.40 \mathrm{M}_{\odot}$.

Figure 4 shows the evolution of the orbital angular velocity $\omega$ versus computational cycle for the first 30,000 code cycles corresponding to $\approx 20$ orbits. The stars were initially placed on the grid using a solution of the TOV equation in isotropic coordinates for an isolated star. The stars were initially set to be corotating but were allowed to settle into their binary equilibrium. Notice that it takes $\sim 5,000$ cycles, corresponding to $\sim 3$ orbits, just to approach the quasiequilibrium binary solution. Indeed, the stars continued to gradually compact and slightly increase in orbital frequency until $\sim 10$ orbits; afterward, the stars were completely stable.

Figure 5 shows the contours of the lapse function $\alpha$ (roughly corresponding to the gravitational potential) and corresponding density profiles at cycle numbers, 0,5200 , and $25800(\approx 0,5$, and 19 orbits). Figure 6 shows the contours of central density and the orientation of the binary orbit corresponding to these cycle numbers. One can visibly see from these figures the relaxation of the stars after the first few orbits and the stability of the density profiles after multiple orbits.

We note, however, that this orbit is on the edge of the ISCO. As such it could be unstable to inspiral even after many orbits. Figures 7 and 8 further illustrate this point. In these simulations various angular momenta were computed with a slightly higher neutron star mass $\left(M_{b}=1.61 \mathrm{M}_{\odot}\right.$ and $M_{g}=1.44 \mathrm{M}_{\odot}$ ), but the same MW EoS. In this case the binary system was followed for nearly 100 orbits.

Figure 7 illustrates orbital angular frequency versus cycle number for three representative angular momenta bracketing the ISCO. The orbital separation for the lowest angular momentum $\left(J=2.7 \times 10^{11} \mathrm{~cm}^{-2}\right)$ shown on Figure 7 is just inside the ISCO. Hence, even though it requires about 10 orbits before inspiral, the orbit is eventually unstable. Similarly, Figure 8 shows the central density versus number of orbits for 6 different angular momenta. Here one can see that orbits with $J \geq 3.0 \times 10^{11} \mathrm{~cm}^{-2}$ are stable. Indeed, for these cases, after about the first 3 orbits the orbits continue with almost no discernible change in orbit frequency or central density.

As mentioned previously, the numerical relativistic neutron binary simulations of [43] all start with initial data that are subsequently evolved in a different manner compared to those with which they were created. One conclusion that may be drawn from the above set of simulations, however, is that the initial data must be evolved for ample time $(>3$ orbit) for the stars to reach a true quasiequilibrium binary configuration. That has not always been done in the literature.

\section{Sensitivity of Initial Condition Orbital Parameters to the Equation of State}

4.1. Equations of State. One hope in the forthcoming detection of gravitational waves is that a sensitivity exists to the neutron star equation of state. For illustration we utilize several representative equations of state often employed in the literature. These span a range from relatively soft to stiff nuclear matter. These are used to illustrate the EoS dependence of the initial conditions. One EoS often employed is that of a polytrope, that is, $p=K \rho^{\Gamma}$, with $\Gamma=2$, where in cgs units, $K=0.0445\left(c^{2} / \rho_{n}\right)$, and $\rho_{n}=2.3 \times 10^{14} \mathrm{~g} \mathrm{~cm}^{-3}$. These parameters, with $\rho_{c}=4.74 \times 10^{14} \mathrm{~g} \mathrm{~cm}^{-3}$, produce an isolated star having a radius $=17.12 \mathrm{~km}$ and baryon mass $=1.5 \mathrm{M}_{\odot}$. As noted in previous sections we utilize the zero temperature and zero neutrino chemical potential MW EoS [32, 53, 65]. The third is the equation of state developed by Lattimer and Swesty [66] with two different choices of compressibility, one having compressibility $K=220 \mathrm{MeV}$ and the other having $K=375 \mathrm{MeV}$. We denote these as LS 220 and LS 375. The fourth EoS has been developed by Glendenning [67]. This EoS has $K=240 \mathrm{MeV}$, which is close to the experimental value [68]. We denote this EoS as GLN. Table 1 illustrates [54] the properties of isolated neutron stars generated with each EoS. For each case the baryon mass was chosen to obtain a gravitational mass for each star of $1.4 \mathrm{M}_{\odot}$.

In Figure 9 we plot the equation of state index $\Gamma$ versus density, $\rho$, for the various EoSs considered here. These are 


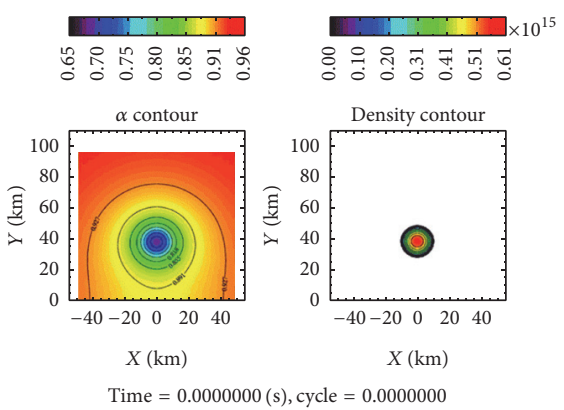

(a)

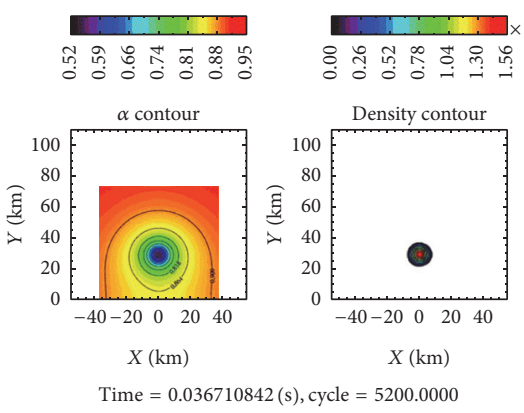

(b)

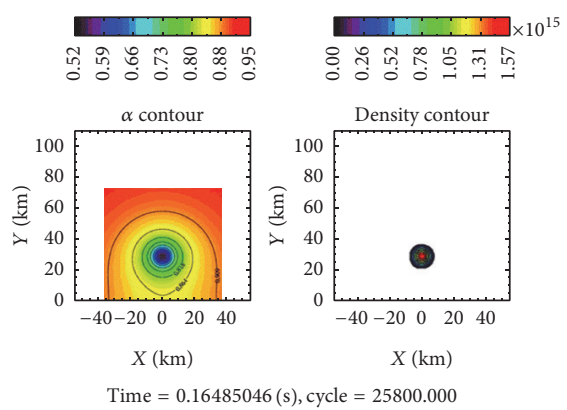

(c)

Figure 5: Contours of the lapse function (left) and central density (right) at cycle numbers 0 (a), 5,200 (b), and 25,800 (c) corresponding to roughly 0,5 , and 19 orbits.
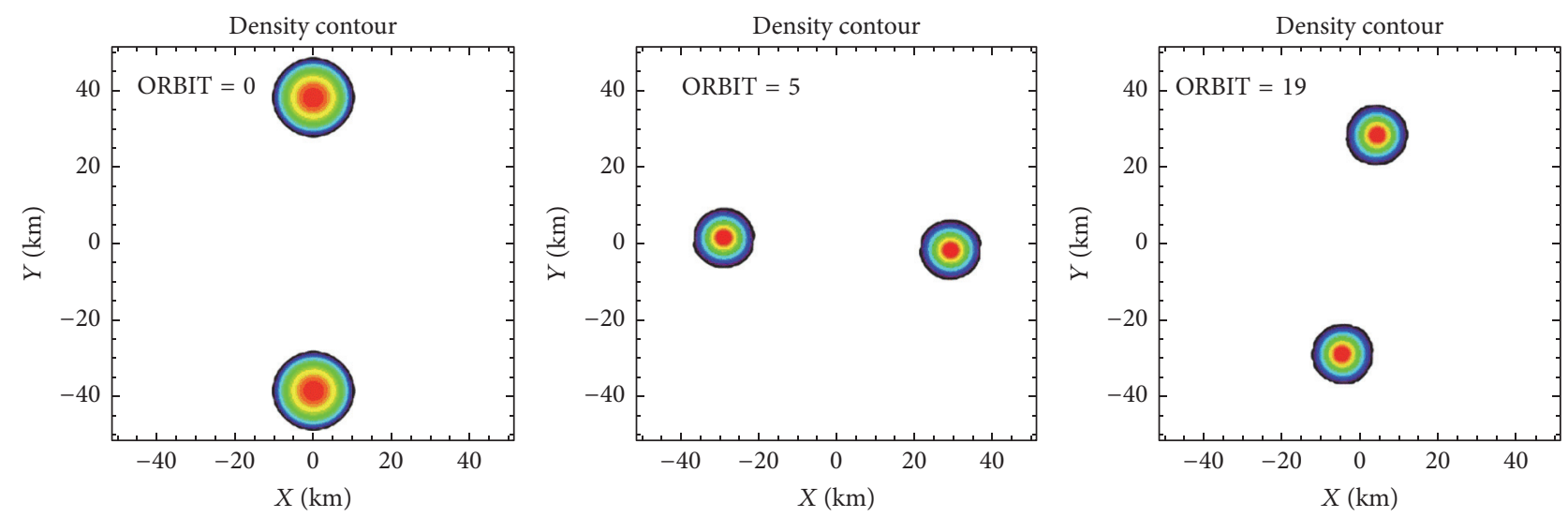

FIGURE 6: Contours of the central density for the binary system at the approximate number of orbits as labelled.

TABLE 1: Table presenting central density, baryon mass, and gravitational mass for the five adopted equations of state.

\begin{tabular}{lccc}
\hline EoS & $\rho_{c}\left(\times 10^{15} \mathrm{~g} \mathrm{~cm}^{-3}\right)$ & $M_{B}\left(\mathrm{M}_{\odot}\right)$ & $M_{G}\left(\mathrm{M}_{\odot}\right)$ \\
\hline GLN & 1.56 & 1.54 & 1.40 \\
MW & 1.39 & 1.54 & 1.40 \\
LS 220 & 0.698 & 1.54 & $\sim 1.40$ \\
LS 375 & 0.492 & 1.54 & $\sim 1.40$ \\
$\Gamma=2$ polytrope & 0.474 & 1.50 & 1.40 \\
\hline
\end{tabular}

compared to the simple polytropic $\Gamma=2$ EoS often employed in the literature.

\subsection{EoS Dependence of the Initial Condition Orbit Parameters.} Table 2 summarizes the initial condition orbit parameters [54] at various fixed angular momenta for the various equations of state. In the case of orbits unstable to merger, this table lists the orbit parameters just before inspiral. These orbits span a range in specific angular momenta $J / M_{0}^{2}$ of $\sim 5$ to 10. The equations of state listed in Tables 1 and 2 are in approximate order of increasing stiffness from the top to the bottom.

As expected, the central densities are much higher for the relatively soft MW and GLN equations of state. Also, the orbit angular frequencies are considerably lower for the extended mass distributions of the stiff equations of state than for the more compact soft equations of state. These extended mass distributions induce a sensitivity of the emergent gravitational wave frequencies and amplitude due to the strong dependence of the gravitational wave frequency to the quadrupole moment of the mass distribution.

4.3. Gravitational Wave Frequency. The physical processes occurring during the last orbits of a neutron star binary are currently a subject of intense interest. As the stars approach their final orbits it is expected that the coupling of the orbital motion to the hydrodynamic evolution of the stars in the strong relativistic fields could provide insight into various physical properties of the coalescing system [58, 69]. In this regard, careful modeling of the initial conditions is needed which includes both the nonlinear general relativistic and hydrodynamic effects as well as a realistic neutron star equation of state.

Figure 10 shows the EoS sensitivity of the gravitational wave frequency $f=\omega / \pi$ as a function of proper separation $d_{p}$ between the stars for the various orbits and equations of state summarized in Table 2. These are compared with the circular orbit condition in the (post) ${ }^{5 / 2}$-Newtonian approximation, hereafter PN, analysis of reference [70]. In that paper 


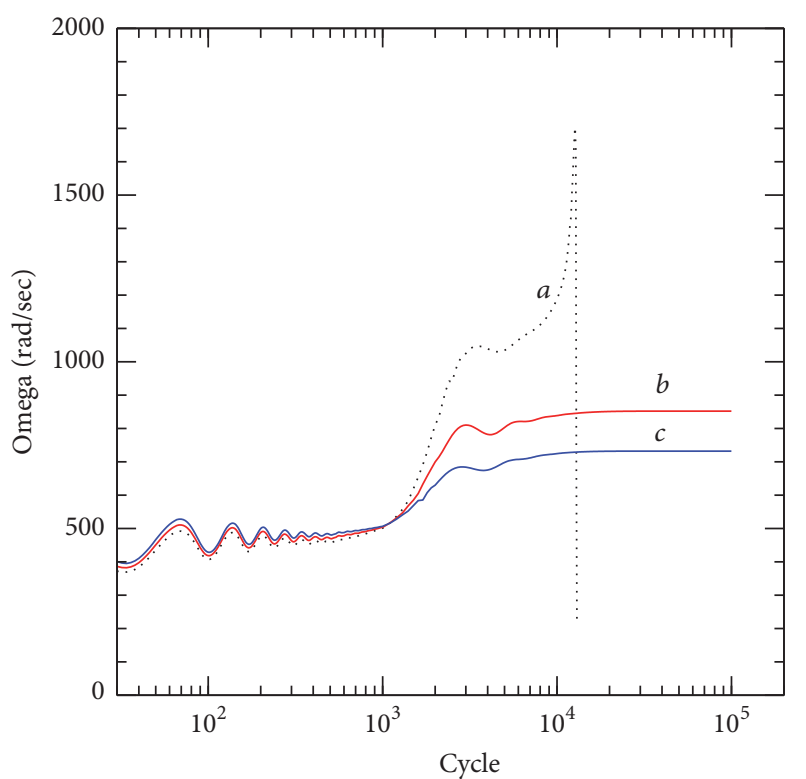

Figure 7: Plot of the orbital angular velocity, $\omega$, versus cycle. When $\omega$ stops changing with time the simulation has reached a circular binary orbit solution. The run $\left(a\right.$ for $J=2.7 \times 10^{11} \mathrm{~cm}^{2}$ ) goes over $\sim 10$ obits and then becomes unstable to inspiral and merger after $\sim 10^{4}$ cycles. The stable two runs ( $b$ for $J=2.8 \times 10^{11} \mathrm{~cm}^{-2}$ and $c$ for $J=2.9 \times 10^{11} \mathrm{~cm}^{-2}$ ) were run for 100,000 cycles and $\approx 100$ orbits.

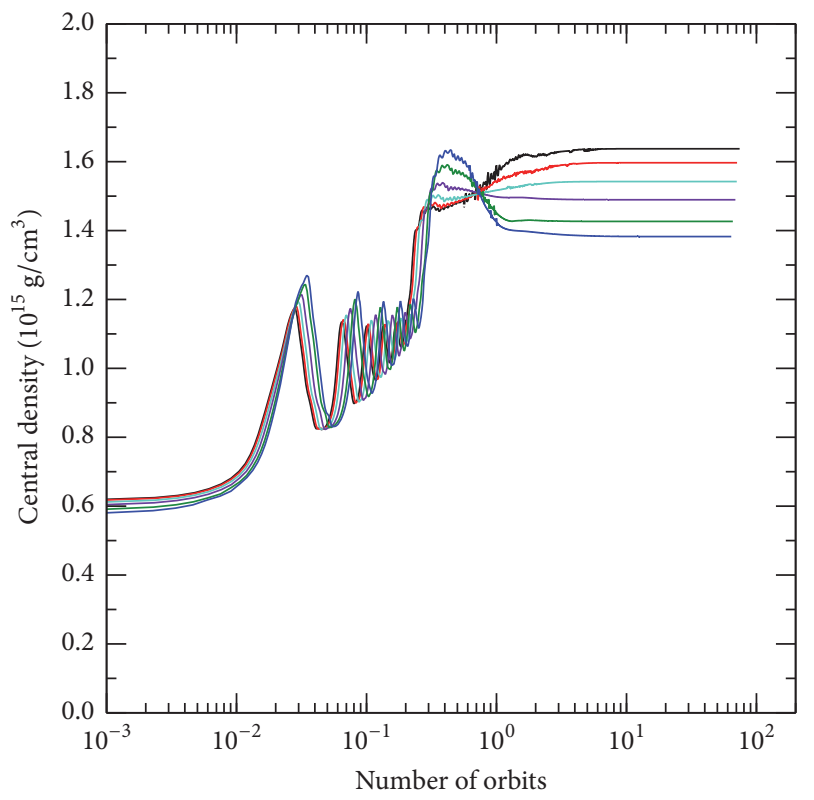

FIgURE 8: Plot of the central density, $\rho_{c}$, versus the number of orbit. The solid lines from top to bottom are for $J=$ $3.0,3.2,3.4,3.6,3.8,4.0 \times 10^{11} \mathrm{~cm}^{2}$.

a search was made for the inner most stable circular orbit in the absence of radiation reaction terms in the equations of motion. This is analogous to the calculations performed here which also analyzes orbit stability in the absence of radiation reaction.

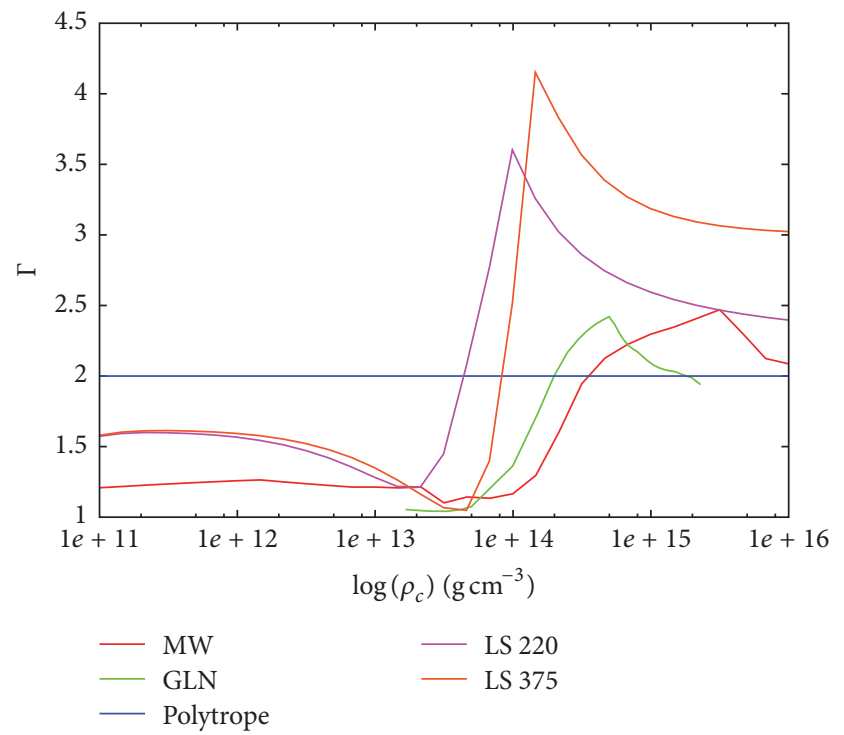

FIGURE 9: EoS index $\Gamma$ versus central density for various equations of state. Large $\Gamma$ implies a stiff EoS.

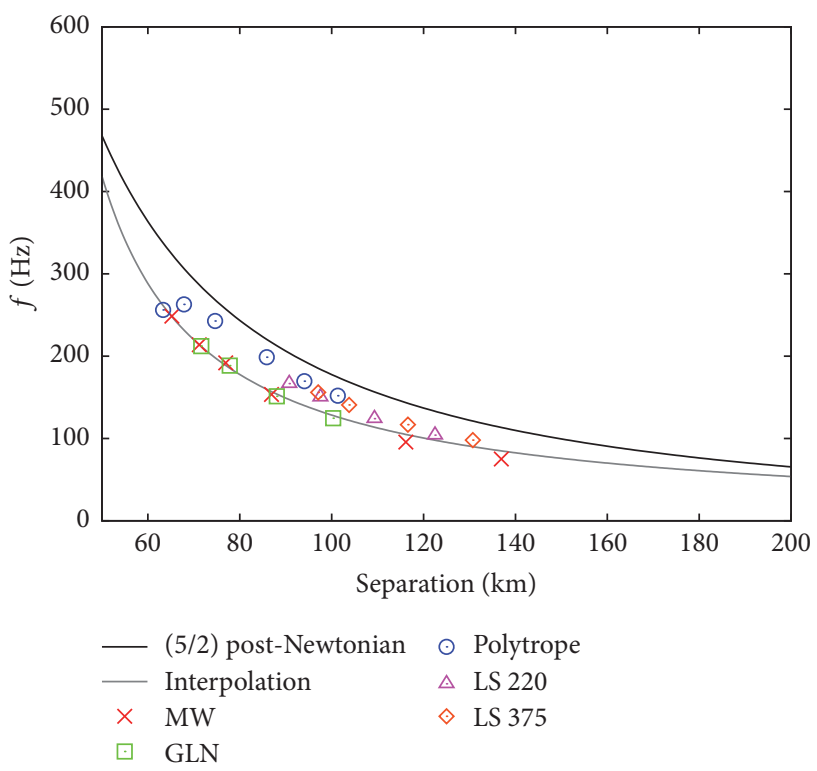

FIGURE 10: Computed gravitational wave frequency, $f$, versus proper separation for each EoS as labelled. The black line corresponds to the (post) ${ }^{5 / 2}$-Newtonian estimate. Frequencies obtained from the stiff and polytropic equations of state do not deviate by more than $\sim 10 \%$ from the PN prediction until a frequency greater than $\sim 300 \mathrm{~Hz}$. The grey line is an extrapolation of the frequencies obtained using the soft MW and GLN EoSs. These begin to deviate by more than $10 \%$ from the PN prediction at a frequency of $\sim 100 \mathrm{~Hz}$.

In the (post) $)^{5 / 2}$-Newtonian equations of motion, a circular orbit is derived by setting time derivatives of the separation, angular frequency, and the radial acceleration to zero. This leads to the circular orbit condition [70]

$$
\omega_{0}^{2}=\frac{m A_{0}}{d_{h}^{3}},
$$


TABLE 2: Orbital parameters for each EoS.

\begin{tabular}{|c|c|c|c|c|c|c|}
\hline EoS & $J\left(\mathrm{~cm}^{2}\right)$ & $\omega\left(\mathrm{rad} \mathrm{s}^{-1}\right)$ & $d_{p}(\mathrm{~km})$ & $d_{c}(\mathrm{~km})$ & $M_{\mathrm{ADM}}\left(\mathrm{M}_{\odot}\right)$ & $\rho_{c}\left(\mathrm{~g} \mathrm{~cm}^{-3}\right)$ \\
\hline \multirow{4}{*}{ GLN } & $2.7 \times 10^{11}$ & 666.5 & 71.62 & 57.67 & 1.390 & $1.73 \times 10^{15}$ \\
\hline & $2.8 \times 10^{11}$ & 592.34 & 77.82 & 62.81 & 1.391 & $1.69 \times 10^{15}$ \\
\hline & $3.0 \times 10^{11}$ & 475.05 & 88.06 & 73.53 & 1.394 & $1.61 \times 10^{15}$ \\
\hline & $3.2 \times 10^{11}$ & 391.75 & 100.34 & 84.31 & 1.396 & $1.56 \times 10^{15}$ \\
\hline \multirow{6}{*}{ MW } & $2.6 \times 10^{11}$ & 780.92 & 65.22 & 51.52 & 1.391 & $1.67 \times 10^{15}$ \\
\hline & $2.7 \times 10^{11}$ & 671.85 & 71.18 & 57.24 & 1.393 & $1.62 \times 10^{15}$ \\
\hline & $2.8 \times 10^{11}$ & 602.80 & 76.94 & 61.86 & 1.394 & $1.60 \times 10^{15}$ \\
\hline & $3.0 \times 10^{11}$ & 482.30 & 86.91 & 72.36 & 1.396 & $1.55 \times 10^{15}$ \\
\hline & $3.5 \times 10^{11}$ & 300.46 & 116.13 & 100.8 & 1.399 & $1.44 \times 10^{15}$ \\
\hline & $3.8 \times 10^{11}$ & 235.72 & 136.93 & 119.74 & 1.401 & $1.39 \times 10^{15}$ \\
\hline \multirow{4}{*}{ LS 220} & $2.7 \times 10^{11}$ & 523.59 & 90.77 & 77.34 & 1.403 & $7.18 \times 10^{14}$ \\
\hline & $2.8 \times 10^{11}$ & 472.08 & 97.53 & 83.08 & 1.404 & $7.14 \times 10^{14}$ \\
\hline & $3.0 \times 10^{11}$ & 389.96 & 109.78 & 94.84 & 1.405 & $7.06 \times 10^{14}$ \\
\hline & $3.2 \times 10^{11}$ & 327.04 & 122.51 & 107.10 & 1.407 & $6.98 \times 10^{14}$ \\
\hline \multirow{4}{*}{ LS 375} & $2.7 \times 10^{11}$ & 490.09 & 97.09 & 83.92 & 1.404 & $5.00 \times 10^{14}$ \\
\hline & $2.8 \times 10^{11}$ & 442.40 & 103.95 & 90.04 & 1.405 & $4.98 \times 10^{14}$ \\
\hline & $3.0 \times 10^{11}$ & 366.67 & 116.65 & 102.50 & 1.406 & $4.95 \times 10^{14}$ \\
\hline & $3.2 \times 10^{11}$ & 307.80 & 130.72 & 115.60 & 1.407 & $4.92 \times 10^{14}$ \\
\hline \multirow{6}{*}{ Polytrope } & $1.8 \times 10^{11}$ & 804.70 & 63.30 & 51.20 & 1.395 & $6.78 \times 10^{14}$ \\
\hline & $2.1 \times 10^{11}$ & 826.03 & 67.85 & 55.18 & 1.396 & $7.00 \times 10^{14}$ \\
\hline & $2.3 \times 10^{11}$ & 762.37 & 74.64 & 61.72 & 1.397 & $6.55 \times 10^{14}$ \\
\hline & $2.5 \times 10^{11}$ & 624.33 & 85.87 & 72.71 & 1.399 & $6.24 \times 10^{14}$ \\
\hline & $2.6 \times 10^{11}$ & 532.83 & 94.04 & 80.45 & 1.400 & $6.17 \times 10^{14}$ \\
\hline & $2.7 \times 10^{11}$ & 477.19 & 101.34 & 86.95 & 1.400 & $6.05 \times 10^{14}$ \\
\hline
\end{tabular}

where $\omega_{0}$ is the circular orbit frequency and $m=2 M_{G}^{0}, d_{h}$ is the separation in harmonic coordinates, and $A_{0}$ is a relative acceleration parameter which for equal mass stars becomes

$$
A_{0}=1-\frac{3}{2} \frac{m}{d_{h}}\left[3-\frac{77}{8} \frac{m}{d_{h}}+\left(\omega_{0} d_{h}\right)^{2}\right]+\frac{7}{4}\left(\omega_{0} d_{h}\right)^{2} .
$$

Equations (26) and (27) can be solved to find the orbit angular frequency as a function of harmonic separation $d_{h}$. The gravitational wave frequency is then twice the orbit frequency, $f=\omega_{0} / \pi$.

Although this is a gauge-dependent comparison, for illustration we show in Figure 10 the calculated gravitational wave frequency compared to the PN expectation as a function of proper binary separation distance up to $200 \mathrm{~km}$. One should keep in mind, however, that there is some uncertainty in associating proper distance with the PN parameter $(\mathrm{m} / \mathrm{r})$. Hence, a comparison with the PN results is not particularly meaningful. It is nevertheless instructive to consider the difference in the numerical results as the stiffness of the EoS is varied. The polytropic and stiff EoSs begin to deviate (by $>10 \%$ ) from the softer equations of state (MW and GLN) for a gravitational wave frequency as low as $\sim 100 \mathrm{~Hz}$ and more or less continue to deviate as the stars approach the ISCO at higher frequencies.

Indeed, a striking feature of Figure 10 is that as the stars approach the ISCO, the frequency varies more slowly with diminishing separation distance for the softer equations of state. A gradual change in frequency can mean more orbits in the LIGO window and hence a stronger signal to noise (cf. [57]).

Also, for a soft EoS the orbit becomes unstable to inspiral at a larger separation. At least part of the difference between the soft and stiff EoSs can be attributed to the effects of the finite size of the stars which are more compact for the soft equations of state [37].

We note that, for comparable angular momenta, our results are consistent with the EoS sensitivity study of [37] based upon a set of equations of state parameterized by a segmented polytropic indices and an overall pressure scale. Their calculations, however, were based upon two independent numerical relativity codes. The similarity of their simulations to the results in Table 2 further confirms the broad validity of the CFA approach when applied to initial conditions. For example, their orbit parameters are summarized in Table II of [37]. Their softest EoS is the Bss221 which corresponds to an adiabatic index of $\Gamma=2.4$ for the core and a baryon mass of $1.501 \mathrm{M}_{\odot}$ and an $\mathrm{ADM}$ mass of $1.338 \mathrm{M}_{\odot}$ per star for a specific angular momentum of $1.61 \times 10^{11} \mathrm{~cm}^{2}$ (in our units) with a corresponding gravitational wave frequency of $530 \mathrm{~Hz}$ at a proper separation of $46 \mathrm{~km}$. This EoS is comparable to the polytropic, MW, and GLN EoSs shown on Figure 10. For example, our closest orbit with the $\Gamma=2$ polytropic EoS corresponds to a specific angular momentum of $1.8 \times 10^{11} \mathrm{~cm}^{2}$ and an ADM mass of $1.39 \mathrm{M}_{\odot}$ compared to their ADM mass 
of $1.34 \mathrm{M}_{\odot}$ at $J=1.6 \times 10^{11} \mathrm{~cm}^{2}$ for the same baryon mass of $1.5 \mathrm{M}_{\odot}$. Although, for the softer EoSs, their results are for a closer orbit than the numerical points given on Figure 10, an extension of the grey line fit to the numerical simulations of the soft EoSs would predict a frequency of $540 \mathrm{~Hz}$ at the same proper separation of $46 \mathrm{~km}$ compared to $530 \mathrm{~Hz}$ in the Bss 221 simulation of [37].

The main parameter characterizing the last stable orbit in the post-Newtonian calculation is the ratio of coordinate separation to total mass (in isolation) $d_{h} / m$. The analogous quantity in our nonperturbative simulation is proper separation to gravitational mass, $d_{P} / m$. The separation corresponding to the last stable orbit in the post-Newtonian analysis does not occur until the stars have approached $6.03 \mathrm{~m}$. For $M_{G}^{0}=1.4 \mathrm{M}_{\odot}$ stars, this would correspond to a separation distance of about $25 \mathrm{~km}$. In the results reported here the last stable orbit occurs somewhere just below $7.7 m_{G}^{0}$ at a proper separation distance of $d_{P} \approx 30 \mathrm{~km}$ for both the polytropic and the MW stars.

\section{Conclusions}

The relativistic hydrodynamic equilibrium in the CFA remains as a viable approach to calculate the initial conditions for calculations of binary neutron stars. In this paper we have illustrated that one must construct initial conditions that have run for at least several orbits before equilibrium is guaranteed. We have demonstrated that beyond the first several orbits the equations are stable over many orbits $(\sim 100)$. We also have shown that such multiple orbit simulations are valuable as a means to estimate the location of the ISCO prior to a full dynamical calculation. Moreover, we have examined the sensitivity of the initial condition orbit parameters and initial gravitational wave frequency to the equation of state. We have illustrated how the initial condition orbital properties (e.g., central densities, orbital velocities, and binding energies) and location of the ISCO are significantly affected by the stiffness of the EoS.

\section{Competing Interests}

The authors declare that there is no conflict of interests regarding the publication of this paper.

\section{Acknowledgments}

The work at the University of Notre Dame (Grant J. Mathews) was supported by the US Department of Energy under Nuclear Theory Grant DE-FG02-95-ER40934 and by the University of Notre Dame Center for Research Computing. One of the authors (N. Q. Lan) was also supported in part by the National Science Foundation through the Joint Institute for Nuclear Astrophysics (JINA) at UND and in part by the Vietnam Ministry of Education (MOE). N. Q. Lan would also like to thank the Yukawa Institute for Theoretical Physics for their hospitality during a visit where part of this work was done.

\section{References}

[1] P. B. Abbot, R. Abbott, T. D. Abbott et al., "Observation of gravitational waves from a binary black hole merger," Physical Review Letters, vol. 116, no. 6, Article ID 061102, 2016.

[2] P. B. Abbot, R. Abbott, T. D. Abbott et al., "GW151226: observation of gravitational waves from a 22-solar-mass binary black hole coalescence," Physical Review Letters, vol. 116, Article ID 241103, 2016.

[3] J. Asai, B. P. Abbott1, R. Abbott et al., "Advanced LIGO," Classical and Quantum Gravity, vol. 32, no. 7, Article ID 074001, 2015.

[4] F. Acernese, M. Agathos, K. Agatsuma et al., "Advanced Virgo: a 2nd generation interferometric gravitational wave detector," Classical and Quantum Gravity, vol. 32, no. 2, Article ID 024001, 2015.

[5] K. Somiya, "Detector configuration of KAGRA-the Japanese cryogenic gravitational-wave detector," Classical and Quantum Gravity, vol. 29, no. 12, 2012.

[6] LIGO Scientific Collaboration, http://www.ligo.org.

[7] K. S. Thorne, "Compact stars in binaries," in Proceedings of the IAU Symposium, J. van Paradijs, E. P. J. van den Heuvel, and E. Kuulkers, Eds., vol. 165, p. 153, Kluwer Academic Publishers, 1996.

[8] G. M. Harry, "Advanced LIGO: the next generation of gravitational wave detectors," Classical and Quantum Gravity, vol. 27, no. 8, Article ID 084006, 2010.

[9] F. A. Rasio and S. L. Shapiro, "Black holes of $D=5$ supergravity," Classical and Quantum Gravity, vol. 16, no. 1, p. 1, 1999.

[10] M. Bailes, "Pulsar velocities," in Proceedings of the IAU Symposium, Compact Stars in Binaries, J. van Paradijs, E. P. J. van den Heuvel, and E. Kuulkers, Eds., vol. 165, p. 213, Hague, the Netherlands, August 1994.

[11] A. V. Tutukov and L. R. YungelSon, "The merger rate of neutron star and black hole binaries," Monthly Notices of the Royal Astronomical Society, vol. 260, no. 3, pp. 675-678, 1993.

[12] E. S. Phinney, "The rate of neutron star binary mergers in the universe-minimal predictions for gravity wave detectors," The Astrophysical Journal, vol. 380, pp. L17-L21, 1991.

[13] V. Kalogera, C. Kim, D. R. Lorimer et al., "The cosmic coalescence rates for double neutron star binaries," The Astrophysical Journal, vol. 601, pp. L179-L182, 2004, Erratum to: The Astrophysical Journal, vol. 614, pp. L137-L138, 2004.

[14] J. Abadie, B. P. Abbott, R. Abbott et al., "Predictions for the rates of compact binary coalescences observable by ground-based gravitational-wave detectors," Classical and Quantum Gravity, vol. 27, no. 17, Article ID 173001, 2010.

[15] M. Burgay, N. D’Amico, A. Possenti et al., "An increased estimate of the merger rate of double neutron stars from observations of a highly relativistic system," Nature, vol. 426, no. 6966, pp. 531533, 2003.

[16] J. M. Lattimer, "The nuclear equation of state and neutron star masses," Annual Review of Nuclear and Particle Science, vol. 62, no. 1, pp. 485-515, 2012.

[17] T. A. Apostolatos, "Construction of a template family for the detection of gravitational waves from coalescing binaries," Physical Review D, vol. 54, no. 4, pp. 2421-2437, 1996.

[18] S. Droz and E. Poisson, "Gravitational waves from inspiraling compact binaries: second post-Newtonian waveforms as search templates," Physical Review D, vol. 56, no. 8, pp. 4449-4454, 1997. 
[19] B. S. Sathyaprakash, "Mother templates for gravitational wave chirps," Classical and Quantum Gravity, vol. 17, no. 23, pp. L157L162, 2000.

[20] A. Buonanno, Y. Chen, and M. Vallisneri, "Detection template families for gravitational waves from the final stages of binaryblack-hole inspirals: nonspinning case," Physical Review D, vol. 67, no. 2, Article ID 024016, 2003.

[21] S. Bose, "Search templates for stochastic gravitational-wave backgrounds," Physical Review D, vol. 71, no. 8, Article ID 082001, 7 pages, 2005.

[22] C. D. Ott, A. Burrows, L. Dessart, and E. Livne, "A New Mechanism for Gravitational-Wave Emission in Core-Collapse Supernovae," Physical Review Letters, vol. 96, no. 20, Article ID 201102, 2006

[23] P. Ajith, N. Fotopoulos, S. Privitera, A. Neunzert, N. Mazumder, and A. J. Weinstein, "Effectual template bank for the detection of gravitational waves from inspiralling compact binaries with generic spins," Physical Review D, vol. 89, no. 8, Article ID 084041, 2014.

[24] F. Pannarale, E. Berti, K. Kyutoku, B. D. Lackey, and M. Shibata, "Gravitational-wave cutoff frequencies of tidally disruptive neutron star-black hole binary mergers," Physical Review D, vol. 92, no. 8, Article ID 081504, 2015.

[25] M. Agathos, J. Meidam, W. Del Pozzo et al., "Constraining the neutron star equation of state with gravitational wave signals from coalescing binary neutron stars," Physical Review DParticles, Fields, Gravitation and Cosmology, vol. 92, no. 2, Article ID 023012, 2015.

[26] J. A. Clark, A. Bauswein, N. Stergioulas, and D. Shoemaker, "Observing gravitational waves from the post-merger phase of binary neutron star coalescence," Classical and Quantum Gravity, vol. 33, no. 8, 2016.

[27] B. Allen, W. G. Anderson, P. R. Brady, D. A. Brown, and J. D. Creighton, "FINDCHIRP: an algorithm for detection of gravitational waves from inspiraling compact binaries," Physical Review D, vol. 85, no. 12, 2012.

[28] L. Blanchet, "Gravitational radiation from post-Newtonian sources and inspiralling compact binaries," Living Reviews in Relativity, vol. 5, article 3, 2002.

[29] L. Blanchet, "Gravitational radiation from post-newtonian sources and inspiralling compact binaries," Living Reviews in Relativity, vol. 17, article no. 2, 2014.

[30] C. K. Mishra, K. Arun, and B. R. Iyer, "Third post-Newtonian gravitational waveforms for compact binary systems in general orbits: instantaneous terms," Physical Review D, vol. 91, no. 8, Article ID 084040, 2015.

[31] J. R. Wilson and G. J. Mathews, "Instabilities in close neutron star binaries," Physical Review Letters, vol. 75, no. 23, article 4161, 1995.

[32] J. R. Wilson, G. J. Mathews, and P. Marronetti, "Relativistic numerical model for close neutron-star binaries," Physical Review D - Particles, Fields, Gravitation and Cosmology, vol. 54, no. 2, article 1317, 1996.

[33] G. J. Mathews, P. Marronetti, and J. R. Wilson, "Relativistic hydrodynamics in close binary systems: analysis of neutron-star collapse," Physical Review D, vol. 58, no. 4, Article ID 043003, 1998.

[34] G. J. Mathews and J. R. Wilson, "Revised relativistic hydrodynamical model for neutron-star binaries," Physical Review D, vol. 61, no. 12, Article ID 127304, 2000.
[35] L. Baiotti, T. Damour, B. Giacomazzo, A. Nagar, and L. Rezzolla, "Analytic modeling of tidal effects in the relativistic inspiral of binary neutron stars," Physical Review Letters, vol. 105, no. 26, Article ID 261101, 2010.

[36] S. Bose, S. Ghosh, and P. Ajith, "Systematic errors in measuring parameters of non-spinning compact binary coalescences with post-Newtonian templates," Classical and Quantum Gravity, vol. 27, no. 11, Article ID 114001, 2010.

[37] J. S. Read, L. Baiotti, J. D. E. Creighton et al., "Matter effects on binary neutron star waveforms," Physical Review D, vol. 88, no. 4, Article ID 044042, 2013.

[38] A. Maselli, L. Gualtieri, and V. Ferrari, "Constraining the equation of state of nuclear matter with gravitational wave observations: tidal deformability and tidal disruption," Physical Review D, vol. 88, no. 10, Article ID 104040, 2013.

[39] A. Bauswein and N. Stergioulas, "Unified picture of the postmerger dynamics and gravitational wave emission in neutron star mergers," Physical Review D, vol. 91, no. 12, Article ID 124056, 2015

[40] A. Bauswein, N. Stergioulas, and H.-T. Janka, "Neutron-star properties from the postmerger gravitational wave signal of binary neutron stars," Physics of Particles and Nuclei, vol. 46, no. 5, pp. 835-838, 2015.

[41] C. L. Fryer, K. Belczynski, E. Ramirez-Ruiz, S. Rosswog, G. Shen, and A. W. Steiner, "The fate of the compact remnant in neutron star mergers," Astrophysical Journal, vol. 812, no. 1, article no. 24, 2015.

[42] T. Dietrich, N. Moldenhauer, N. K. Johnson-McDaniel et al., "Binary neutron stars with generic spin, eccentricity, mass ratio, and compactness: quasi-equilibrium sequences and first evolutions," Physical Review D-Particles, Fields, Gravitation and Cosmology, vol. 92, no. 12, Article ID 124007, 2015.

[43] M. D. Duez, P. Marronetti, S. L. Shapiro, and T. W. Baumgarte, "Hydrodynamic simulations in $\mathbf{3}+\mathbf{1}$ general relativity," Physical Review. D. Third Series, vol. 67, no. 2, 024004, 22 pages, 2003.

[44] P. Marronetti, M. D. Duez, S. L. Shapiro, and T. W. Baumgarte, "Dynamical determination of the innermost stable circular orbit of binary neutron stars," Physical Review Letters, vol. 92, no. 14, Article ID 141101, 2004.

[45] M. Miller, P. Gressman, and W. Suen, "Towards a realistic neutron star binary inspiral: initial data and multiple orbit evolution in full general relativity," Physical Review D, vol. 69, no. 6, Article ID 064026, 2004.

[46] M. Miller, "Accuracy requirements for the calculation of gravitational waveforms from coalescing compact binaries in numerical relativity," Physical Review D, vol. 71, no. 10, Article ID 104016, 2005.

[47] M. Miller, "General-relativistic decompression of binary neutron stars during dynamic inspiral," Physical Review D, vol. 75, no. 2, Article ID 024001, 2007.

[48] K. Uryu, F. Limousin, J. L. Friedman, E. Gourgoulhon, and M. Shibata, "Binary neutron stars: equilibrium models beyond spatial conformal flatness," Physical Review Letters, vol. 97, no. 17, Article ID 171101, 2006.

[49] K. Kiuchi, Y. Sekiguchi, M. Shibata, and K. Taniguchi, "Longterm general relativistic simulation of binary neutron stars collapsing to a black hole," Physical Review D, vol. 80, no. 6, Article ID 064037, 2009.

[50] S. Bernuzzi, A. Nagar, T. Dietrich, and T. Damour, "Modeling the dynamics of tidally interacting binary neutron stars up to the merger," Physical Review Letters, vol. 114, no. 16, Article ID 161103, 2015. 
[51] R. De Pietri, A. Feo, F. Maione, and F. Löffler, "Modeling equal and unequal mass binary neutron star mergers using public codes," Physical Review D, vol. 93, no. 6, Article ID 064047, 2015.

[52] É. É. Flanagan, "Possible explanation for star-crushing effect in binary neutron star simulations," Physical Review Letters, vol. 82, no. 7, pp. 1354-1357, 1999.

[53] J. R. Wilson and G. J. Mathews, Relativistic numerical hydrodynamics, Cambridge Monographs on Mathematical Physics, Cambridge University Press, Cambridge, UK, 2003.

[54] J. R. Haywood, Numerical relativistic hydrodynamic simulations of neutron stars [Ph.D. thesis], University of Notre Dame, 2006.

[55] E. Gourgoulhon, P. Grandclément, K. Taniguchi, J. Marck, and S. Bonazzola, "Quasiequilibrium sequences of synchronized and irrotational binary neutron stars in general relativity: method and tests," Physical Review D, vol. 63, no. 6, Article ID 064029, 2001.

[56] K. Taniguchi, E. Gourgoulhon, and S. Bonazzola, "Quasiequilibrium sequences of synchronized and irrotational binary neutron stars in general relativity. II. Newtonian limits," Physical Review D, vol. 64, Article ID 064012, 2001.

[57] N. Q. Lan, I.-S. Suh, G. J. Mathews, and J. R. Haywood, "Gravitational waveforms from multiple-orbit simulations of binary neutron stars," Communications in Physics, vol. 25, no. 4, pp. 299-308, 2015.

[58] G. J. Mathews and J. R. Wilson, "Binary-induced neutron star compression, heating, and collapse," The Astrophysical Journal, vol. 482, no. 2, pp. 929-941, 1997.

[59] R. Arnowitt, S. Deser, and C. W. Misner, "Republication of: the dynamics of general relativity," General Relativity and Gravitation, vol. 40, no. 9, pp. 1997-2027, 2008.

[60] J. W. York Jr. and L. Smarr, Sources of Gravitational Radiation, Cambridge University Press, Cambridge, UK, 1979.

[61] K. S. Thorne, "Multipole expansions of gravitational radiation," Reviews of Modern Physics, vol. 52, no. 2, pp. 299-339, 1980.

[62] C. R. Evans, A method for numerical relativity: simulation of axisymmetric gravitational collapse and gravitational radiation generation [Ph.D. thesis], University of Texas at Austin, 1984.

[63] J. R. Wilson, Sources of Gravitational Radiation ed Smarr L L, Cambridge University Press, Cambridge, UK, 1979.

[64] C. W. Misner, K. S. Thorne, and J. A. Wheeler, Gravitation, W. H. Freeman and Co., San Francisco, Calif, USA, 1973.

[65] R. W. Mayle, M. Tavani, and J. R. Wilson, "Pions, supernovae, and the supranuclear matter density equation of state," Astrophysical Journal, vol. 418, no. 1, pp. 398-404, 1993.

[66] J. M. Lattimer and F. D. Swesty, "A generalized equation of state for hot, dense matter," Nuclear Physics A, vol. 535, no. 2, pp. 331376, 1991.

[67] N. K. Glendenning, Compact Stars: Nuclear Physics, Particle Physics, and General Relativity, Springer, New York, NY, USA, 1996.

[68] U. Garg, "The isoscalar giant dipole resonance: a status report," Nuclear Physics A, vol. 731, pp. 3-14, 2004.

[69] C. Cutler, T. A. Apostolatos, L. Bildsten et al., "The last three minutes: issues in gravitational-wave measurements of coalescing compact binaries," Physical Review Letters, vol. 70, no. 20 , article $2984,1993$.

[70] L. E. Kidder, C. M. Will, and A. G. Wiseman, "Spin effects in the inspiral of coalescing compact binaries," Physical Review D, vol. 47, no. 10, pp. R4183-R4187, 1993. 

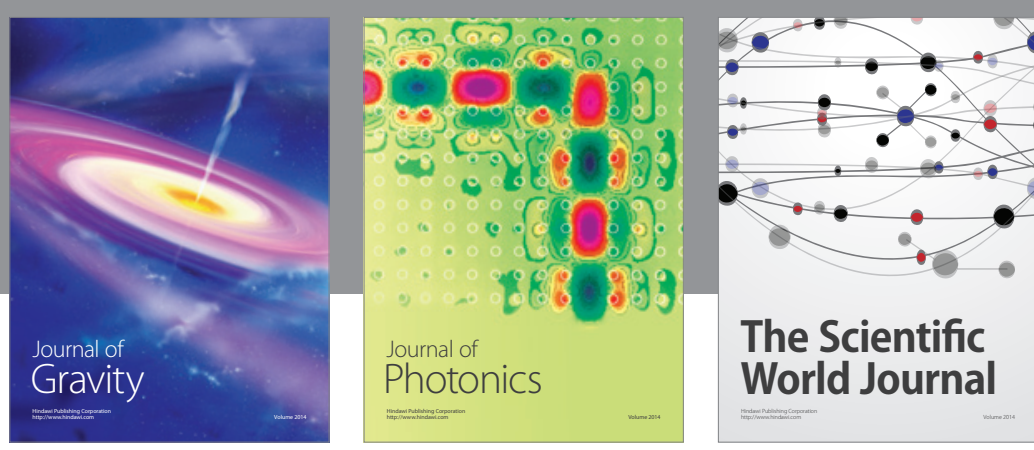

The Scientific World Journal
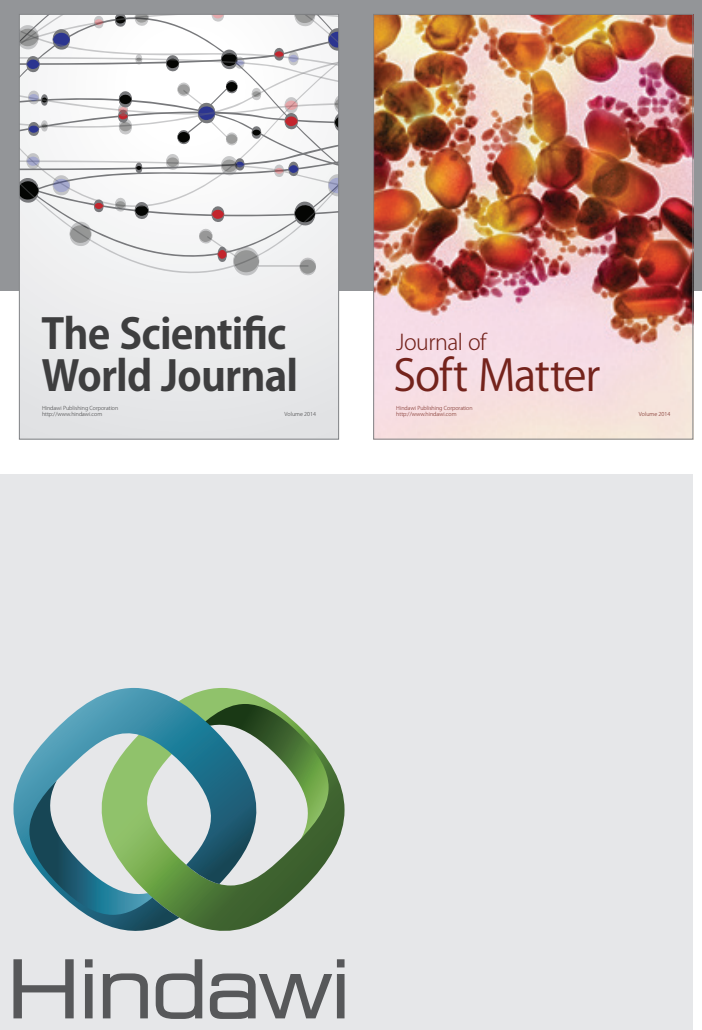

Submit your manuscripts at

https://www.hindawi.com
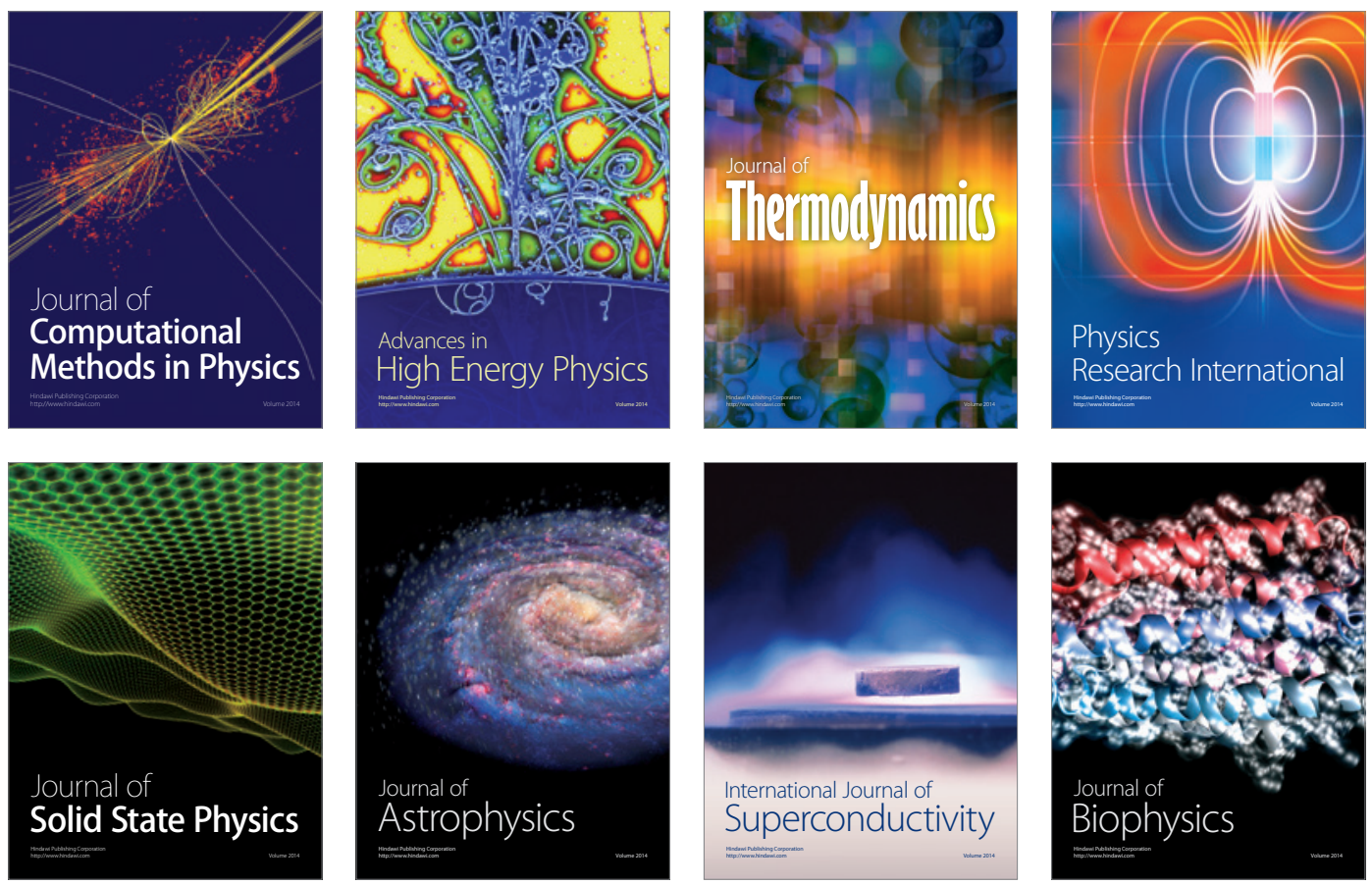
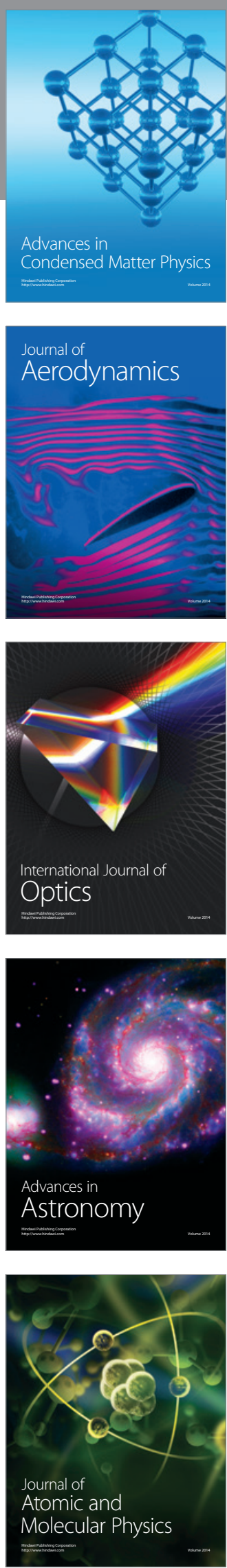